\title{
A Comparison of Methods Used for Quantifying Internal Training Load in Women Soccer Players
}

\author{
Helen Alexiou and Aaron J. Coutts
}

\begin{abstract}
Purpose: The purpose of this study was to compare the session-RPE method for quantifying internal training load (TL) with various HR-based TL quantification methods in a variety of training modes with women soccer players. Methods: Fifteen elite women soccer players took part in the study (age: $19.3 \pm 2.0$ y and $\mathrm{V}_{\text {O2max }}: 50.8$ $\left.\pm 2.7 \mathrm{~mL} \cdot \mathrm{kg}^{-1} \cdot \mathrm{min}^{-1}\right)$. Session-RPE, heart rate, and duration were recorded for 735 individual training sessions and matches over a period of $16 \mathrm{wk}$. Correlation analysis was used to compare session-RPE TLs with three commonly used HR-based methods for assessing TL. Results: The mean correlation for session-RPE TL with Banister's TRIMP, $\mathrm{LT}_{\text {zone }} \mathrm{TL}$ and Edwards's TL were $(r=0.84,0.83$, and 0.85 , all $P<.01$, respectively). Correlations for session-RPE TL and three HR-based methods separated by session type were all significant (all $P<.05$ ). The strongest correlations were reported for technical ( $r=0.68$ to 0.82 ), conditioning ( $r=0.60$ to 0.79 ), and speed sessions ( $r=0.61$ to 0.79 ). Conclusion: The session-RPE TL showed a significant correlation with all training types common to soccer. Higher correlations were found with less intermittent, aerobic-based training sessions and suggest that HR-based TLs relate better to session-RPE TLs in less intermittent training activities. These results support previous findings showing that the session-RPE TL compares favorably with HR-based methods for quantifying internal TL in a variety of soccer training activities.
\end{abstract}

Keywords: session-RPE, heart rate, soccer training, periodization

To optimize athletic performance, physical training should be prescribed to suit each athlete's individual characteristics. However, in team sports like soccer, training sessions are often conducted in a group, which reduces the likelihood that players are receiving specific training based on their individual characteristics. ${ }^{1}$ For example, Hoff et al, ${ }^{2}$ demonstrated that soccer players with superior fitness levels did not receive sufficient training stimulus to further increase their fitness when training in a team environment using small-sided games alone. It has also been suggested that players with inferior fitness levels may be overstressed during

The authors are with the School of Leisure, Sport, and Tourism, University of Technology, Sydney, Australia. 
team-based training sessions leading to increased fatigue, injury, and a reduction in performance. ${ }^{1}$ Collectively, these findings show that individuals within the same soccer team may not receive an appropriate level of training stimulus when a team-based training approach is undertaken. Therefore, to overcome the limitations associated with team-based training, it has been suggested that a simple system that quantifies an individual's response to training (ie, internal TL) is developed, so that coaches can monitor and modify training according to individual players' needs.

The assessment of internal TL requires quantification of the intensity and duration of the physiological stress imposed on the athlete. ${ }^{3}$ While the duration of the training session is simple to measure, exercise intensity is more difficult to quantify. However, the most common methods used to measure exercise intensity in soccer are heart rate (HR) and ratings of perceived exertion (RPE). ${ }^{1,4}$ The use of HR to measure exercise intensity is based on the well-known linear relationship between $\mathrm{HR}$ and $\mathrm{V}_{\mathrm{O} 2 \max }$ over a wide range of steady-state submaximal workloads. ${ }^{5}$ However, there are several limitations associated with HR-based methods for quantifying internal TL. For example, a high level of technical expertise is required to collect and collate HR information from a whole team, collecting and analyzing HR data for each player can be time consuming, there is a chance for technical errors, and the financial cost associated with purchasing and maintaining telemetric HR systems can be high. Finally, another limitation of HR-based methods for quantifying internal TL in soccer is that it is a relatively poor method of evaluating very high intensity (and/or short duration) exercise such as resistance training, high intensity interval training, and plyometric training. ${ }^{6}$ For these reasons the use of HR-based methods for quantifying TL may not be the most valid or practical approach for measuring TL in the field.

Foster $^{3}$ proposed an alternative method for assessing internal TL utilizing Borg's Category Ratio-10 (CR-10) RPE scale as a measure of exercise intensity. Using this method, internal TL can be calculated by multiplying the training duration by the rating of perceived exertion (RPE) score. While this method was originally proposed for endurance athletes, research has recently shown that this method has a good level of agreement with HR-based methods for quantifying TL in team sports ${ }^{7}$ and in particular soccer players. ${ }^{6}$ However, a limitation of previous studies examining session-RPE is that the majority of them have focused on endurance sports or one type of training mode (ie, resistance training) ${ }^{8-10}$ Moreover, the few studies that have focused on team sports have established the validity of the session-RPE during aerobic interval training using small-sided soccer games training. Therefore, this study will assess the validity of the session-RPE method across all the training types typical of a soccer training program, therefore including both aerobic and technical/tactical training as well as anaerobic training and matches.

The session-RPE method may provide valuable information in regards to monitoring TL throughout the season and appears to be of great benefit for monitoring individual soccer players' TL. As well as providing valuable information in regards to monitoring TL, the session-RPE method provides a practical alternative to using HR-based methods. However, the relationship between session-RPE derived TLs and HR-based TLs across a range of exercise types in women soccer 
players has not been fully assessed. Therefore, the purpose of this study is to compare the session-RPE method with various HR-based methods for quantifying internal TL with women soccer players in a variety of training modes.

\section{Methods}

\section{Subjects}

Fifteen elite women soccer players (age: $19.3 \pm 2.0$ years, height: $169.0 \pm 5.1 \mathrm{~cm}$, body mass: $64.8 \pm 7.7 \mathrm{~kg}, \mathrm{~V}_{\mathrm{O} 2 \max }: 50.8 \pm 2.7 \mathrm{~mL} \cdot \mathrm{kg}^{-1} \cdot \mathrm{min}^{-1}$ ) were recruited for this study. All were scholarship holders at the Football Association (FA) National Player Development Centre (Loughborough University, Loughborough, UK). Ten of the fifteen subjects were members of the England international age-group team at Under 17 years, Under 19 years, Under 21 years, or open age level. Before the commencement of this study all subjects were given an information sheet outlining potential risks associated with involvement in this study. A written consent form was also obtained from each subject or their parent. Before any testing ethical approval was granted by an Institutional Human Research Ethics Committee.

\section{Study Design}

The data for this study was collected from 15 high performance women soccer players over a 16-week soccer season. All subjects completed a maximum oxygen uptake test $\left(\mathrm{V}_{\mathrm{O} 2 \max }\right)$ and a lactate threshold (LT) test at the beginning of the competitive season to determine the individual HR training zones. Heart rate and session-RPE were monitored during each training session and match during the season. The relationships between the session-RPE TL and commonly used HRbased TL quantification methods were used to examine the criterion validity of the session-RPE. The strength of the relationship was reported within each individual player and also for each different training type completed during the season.

\section{Physical Training}

The training program was set by the players' coaching panel throughout the study period. Each player usually completed eight training sessions per week during the study period. The typical training week consisted of three technical/tactical sessions, two high-intensity resistance training sessions, one aerobic conditioning session, one core stability session, one pool "recovery" session, and a competitive match. The training sessions were usually conducted together with the entire training squad. The technical/tactical sessions usually focused on acquisition and refinement of soccer-specific skills. The resistance training sessions were usually involved 6 to 10 exercises of 6 to 12 repetitions at various lifting speeds. Depending on the focus of training and each player's individual needs, from one to three sets of each resistance training exercise were completed with $30 \mathrm{~s} / 3$ min rest between each set. The core stability sessions focused on developing core body strength and posture and involved resistance training exercises. The pool sessions were completed within $24 \mathrm{~h}$ following matches and were of low intensity and continuous in nature. The aerobic conditioning sessions involved either 
high-intensity, small-sided soccer games or high-intensity interval running training. The competitive soccer matches were played according to the normal Fédération Internationale de Football Association regulations.

The TL for each session was calculated using the session-RPE method $^{7}$ for each player during the study period. This method involved multiplying the training duration in minutes by the mean training intensity. ${ }^{7}$ The training intensity was measured using a modified 10-point Rating of Perceived Exertion Scale (CR-10: RPE $)^{11}$ shown in Table 1 . To ensure the subjects reported a mean RPE for the entire training session, the RPE was taken 30 minutes after the completion of the session using previously described methods. ${ }^{6}$ The TL for each day of the week was summed to provide a weekly TL. All data were entered into an online database for analysis of team and individual TLs (www.trainingload.com, Acceleration Australia, Brisbane).

Training intensity for each player was also recorded continuously throughout each training session using Polar HR monitors (Polar Oy, Finland). The HR data were recorded every $5 \mathrm{~s}$. To reduce HR recording error during training, all subjects were asked to check their HR monitors before each session and after each set $(\sim 10$ min). Following each training session, HR information was then downloaded to a computer using Polar Advantage Software.

Several HR-based methods for quantifying TL were used as the criterion measure of internal TL in this investigation. The TRIMP method proposed by Banister et $\mathrm{al}^{12}$ assumes each exercise bout elicits a training impulse. The expression of TL measured in TRIMP units is determined using the following formula:

$$
\mathrm{TRIMP}=\mathrm{D}(\Delta \mathrm{HR} \text { ratio }) \mathrm{e}^{\mathrm{b}(\Delta \mathrm{HR} \text { ratio })}
$$

where $\mathrm{D}=$ duration of training session and $\mathrm{b}=1.67$ for females and 1.92 for males. ${ }^{13}$

\section{Table 1 The Borg Category Ratio-10 Rating of Perceived Exertion Scale ${ }^{8}$}

\begin{tabular}{ll}
\hline Rating & Description \\
\hline 0 & Rest \\
1 & Very, Very Easy \\
2 & Easy \\
3 & Moderate \\
4 & Somewhat Hard \\
5 & Hard \\
6 & \\
7 & Very Hard \\
8 & \\
9 & \\
10 & Maximal \\
\hline
\end{tabular}




\section{$\Delta$ HR ratio $=($ HRex - HRrest $) /($ HRmax - HRrest $)$}

where HRrest $=$ the average heart rate during rest and HRex $=$ the average HR during exercise. The player's HRrest was measured during 5 min of seated rest every morning during the study period.

The HR-based method proposed by Edwards ${ }^{14}$ for determining internal TL was also used as another criterion measure of internal TL in this study. The Edwards ${ }^{14} \mathrm{HR}$-based method involved integrating the total volume of the training session with the total intensity of the exercise session relative to five intensity phases. An exercise score for each training bout was calculated by multiplying the accumulated duration in each HR zone by a multiplier allocated to each zone ( $50 \%$ to $60 \% \mathrm{HRmax}=1,60 \%$ to $70 \% \mathrm{HR} \max =2,70 \%$ to $80 \% \mathrm{HRmax}=3,80 \%$ to $90 \%$ HRmax $=4$, and $90 \%$ to $100 \%$ HRmax $=5$ ) and then summating the results.

The final criterion measure of internal TL used in this study was the HRbased approach based on lactate thresholds $\left(\mathrm{LT}_{\text {zone }}\right)$. This approach has previously been used in a similar investigation. ${ }^{6}$ This method involves multiplying the time spent in three heart rate zones (zone 1: below lactate threshold (LT), zone 2: between LT and the anaerobic threshold (AT); and zone 3: above AT) by a coefficient relative to each intensity zone $(\mathrm{k}=1$ for zone $1, \mathrm{k}=2$ for zone 2 , and $\mathrm{k}=3$ for zone 3 ) and then summating the results. The main difference between the Edwards and $\mathrm{LT}_{\text {zone }}$ methods for quantifying TL are that the HR zones determined in Edwards' $\mathrm{s}^{14}$ investigation are based on standardized predetermined zones, whereas $\mathrm{LT}_{\text {zone }} \mathrm{HR}$ zones are based on individual parameters determined in the laboratory.

Session-RPE and HR-based TL data were collected for 735 individual training sessions and matches. For the lactate threshold heart rate zone $\left(\mathrm{LT}_{\text {zone }}\right) \mathrm{TL}$ method, only 623 of the total training samples and matches were used, because LT data were not collected from three players. For each player, a minimum of 20 sessions of RPE and HR-based TL data were used to ensure adequate statistical power with the correlation analysis.

\section{Physiological Tests}

All subjects completed a maximal oxygen uptake test and twelve players completed a lactate threshold test.

Lactate threshold was measured using an incremental run on a motorized treadmill (RunRace, Technogym, Gambettola, Italy) in an environmentally controlled human performance laboratory. The test began at between 8 and $9 \mathrm{~km} \cdot \mathrm{h}^{-1}$ dependant on individual. Treadmill speed was increased every four minutes by 1 $\mathrm{km} \cdot \mathrm{h}^{1}$ until $12 \mathrm{~km} \cdot \mathrm{h}^{-1}$, and then by $0.5 \mathrm{~km} \cdot \mathrm{h}^{-1}$ for the final four-minute stages. Blood samples $(25 \mu \mathrm{L})$ were taken at rest, along with two samples collected toward the end of each stage from the players thumb and analyzed using a YSI 2300 STAT Plus Lactate Analyser (Fleet, Hampshire, England). Lactate threshold was determined using the $\mathrm{D}_{\max }$ method with the Lactate-E macro add-in ${ }^{15}$ for Microsoft Excel (Microsoft Corporation, USA).

To determine maximum oxygen uptake, the players completed an uphill incremental treadmill run to exhaustion ${ }^{16}$ on a motorized treadmill (RunRace, 
Technogym, Gambettola, Italy). The test began with a 5-minute warm-up at 8 $\mathrm{km} \cdot \mathrm{h}^{-1}$. The treadmill speed was kept constant throughout the test and the inclination was increased from an initial gradient of $3.5 \%$ by $2.5 \%$ every $3 \mathrm{~min}$. Expired air samples were collected during the 1:45 to 2:45 minutes of each 3-minute stage. A final expired air sample was taken during the last minute of the test, immediately after the player signaled that the running speed could be maintained for one final minute. The highest value for oxygen uptake, collected over a 60-s period, during this test was considered to be the $\mathrm{V}_{\mathrm{O} 2 \max }$ of the participant. ${ }^{17}$ Heart rate was recorded throughout the incremental test using portable HR monitors (Polar NV HR monitor). Maximal oxygen uptake was measured using the conventional Douglas bag method. Expired gases were analyzed using Servomex 1440 (Cowborough, Sussex, England), which was calibrated before each test with reference and calibration gases of known concentrations and a Harvard Dry Gas Meter (Edenbridge, Kent, UK).

\section{Statistical Analyses}

The relationship between session-RPE and previously used HR-based methods for monitoring TL were analyzed using Pearson's product-moment correlation. Relationships were determined between each of these methods for a) each session completed by each individual player and b) each type of training completed by the players. Differences between the mean TL for each exercise type were determined using a one-way ANOVA with a Scheffe post hoc test. The mean, standard deviation (SD), and $95 \%$ confidence intervals (CI) were also calculated for the group data. Statistical significance was set at $P<.05$. SPSS statistical software package version 11.5 (SPSS Inc., Chicago, USA) was used for all statistical calculations.

\section{Results}

Individual correlations of session-RPE and all three HR-based TL methods are outlined in Table 2 . All correlations were statistically significant $(P<.01)$. There were also significant correlations between the session-RPE and all the HR-based TL methods for each of the various training modalities (Table 3). The correlation analysis for the various training modes demonstrated that approximately half the variance in the HR-based training methods could be accounted for by the sessionRPE TL for the Conditioning (Banister's TL-55\%; LT $_{\text {zone }}$ TL-35\%; Edwards's

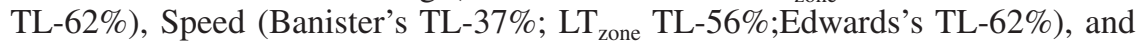
Technical (Banister's TL-46\%; LT $_{\text {zone }}$ TL-48\%; Edwards's TL-67\%) training sessions. However, the session-RPE TL accounted for less of the variance in the HR-based TLs for the Matches (Banister's TL-24\%; LT $_{\text {zone }}$ TL-24\%; Edwards's TL-41\%) and Resistance (Banister's TL-6\%; LT $_{\text {zone }}$ TL-12\%; Edwards's TL-27\%) training sessions.

Figure 1 shows the mean $( \pm \mathrm{SD})$ session TL for the session-RPE and HRbased TL methods for each of the common training modalities completed during the study periods. A one-way analysis of variance demonstrated that the TLs for each monitoring method (ie, Banister's TRIMP, LT $_{\text {zone }}$ TL, and Edwards's TL) was not significantly different $(F=0.15$, df $=2, P=.86)$. However, within ses- 
Table 2 Individual Correlations Between Session-RPE TL and Three HR-Based TL Methods

\begin{tabular}{lcccc}
\hline Subject & $\mathbf{N}$ & $\begin{array}{c}\text { Banister's } \\
\text { TRIMP }\end{array}$ & $\begin{array}{c}\text { LT }_{\text {zone }} \\
\text { TL }\end{array}$ & $\begin{array}{c}\text { Edwards's } \\
\text { TL }\end{array}$ \\
\hline 1 & 20 & 0.75 & 0.88 & 0.50 \\
2 & 115 & 0.76 & 0.82 & 0.74 \\
3 & 20 & 0.92 & 0.85 & 0.93 \\
4 & 60 & 0.91 & 0.63 & 0.93 \\
5 & 26 & 0.76 & $--^{\mathrm{a}}$ & 0.85 \\
6 & 80 & 0.88 & 0.93 & 0.95 \\
7 & 39 & 0.74 & 0.56 & 0.78 \\
8 & 20 & 0.95 & 0.66 & 0.91 \\
9 & 20 & 0.94 & 0.97 & 0.96 \\
10 & 58 & 0.81 & 0.88 & 0.91 \\
11 & 80 & 0.91 & 0.94 & 0.93 \\
12 & 42 & 0.67 & $-{ }^{\mathrm{a}}$ & 0.72 \\
13 & 39 & 0.87 & 0.90 & 0.90 \\
14 & 44 & 0.89 & $-{ }^{\mathrm{a}}$ & 0.89 \\
15 & 72 & 0.90 & 0.94 & 0.92 \\
& & & & $0.56-0.97$ \\
\hline Range & & $0.67-0.95$ & $0.83 \pm 0.14$ & $0.85 \pm 0.12$ \\
Mean \pm SD & & $0.84 \pm 0.09$ & $(0.74-0.92)$ & $(0.79-0.92)$ \\
\hline
\end{tabular}

${ }^{a} \mathrm{LT}_{\text {zone }} \mathrm{TL}$ was not calculated because lactate threshold measurements were not available. All correlations were significant $(P<.01)$.

sion-RPE and each monitoring method highly significant differences were detected based on training type $(P<.001)$. A post hoc analysis showed that match loads were significantly greater than resistance training, speed, and conditioning, and that resistance training were less than technical sessions.

\section{Discussion}

This study is the first to our knowledge to compare session-RPE derived TLs with various HR-based TLs in a variety of training types in elite women soccer players. The results showed that the session-RPE method had a significant positive correlation with three HR-based methods for quantifying TL within each of the 15 players examined. Moreover, we also found significant positive correlations between session-RPE TL and the various HR-based TL methods across various training activities common to soccer. The present findings support previous studies reporting the session-RPE as a practical tool for monitoring internal TL in a variety of training activities. ${ }^{6,7}$ 


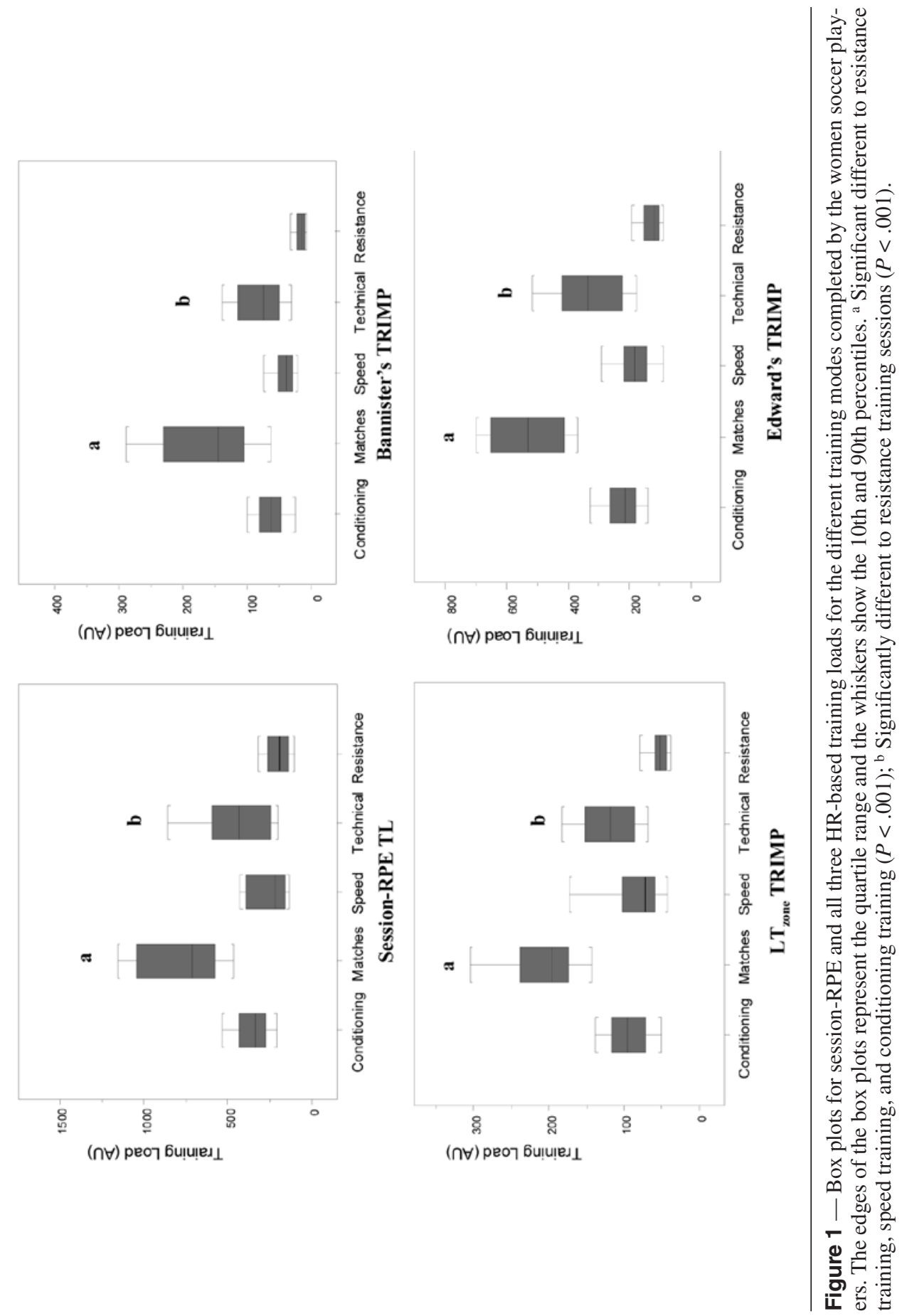


Table 3 Correlation Coefficients for Session-RPE TL and Three HRBased TL Methods Separated by Session Type for the Combined Group of Players

\begin{tabular}{|c|c|c|c|c|c|c|c|c|c|}
\hline & \multicolumn{3}{|c|}{ Bannister's TRIMP } & \multicolumn{3}{|c|}{$\mathrm{LT}_{\text {zone }} \mathrm{TL}$} & \multicolumn{3}{|c|}{ Edwards's TL } \\
\hline & $r$ & $\mathbf{N}$ & $\mathbf{P}$ & $r$ & $\mathbf{N}$ & $\mathbf{P}$ & $r$ & $\mathbf{N}$ & $\mathbf{P}$ \\
\hline Conditioning & 0.74 & 139 & $<0.001$ & 0.60 & 119 & $<0.001$ & 0.79 & 139 & $<0.001$ \\
\hline Matches & 0.49 & 65 & $<0.001$ & 0.49 & 56 & $<0.001$ & 0.64 & 65 & $<0.001$ \\
\hline Speed & 0.61 & 59 & $<0.001$ & 0.75 & 48 & $<0.001$ & 0.79 & 59 & $<0.001$ \\
\hline Technical & 0.68 & 230 & $<0.001$ & 0.69 & 200 & $<0.001$ & 0.82 & 230 & $<0.001$ \\
\hline Resistance & 0.25 & 242 & $<0.001$ & 0.34 & 200 & $<0.001$ & 0.52 & 242 & $<0.001$ \\
\hline
\end{tabular}

In agreement with previous research, ${ }^{6,7}$ we observed moderate-to-strong relationships between session-RPE TL and HR-based TL methods ( $r=0.56$ to 0.97$)$ within the individual players. The mean correlations between the HR-based TL methods and RPE within individual players in this study (0.83 to 0.85 ) are higher than those reported in previous studies that examined soccer training ${ }^{6}$ but lower than studies that have examined steady-state exercise. ${ }^{18}$ A possible explanation for the lower correlations in this study compared with other similar research that involved more steady-state exercise could be attributed to an increased anaerobic contribution due to the stochastic nature of work in soccer training. ${ }^{4}$ In support of this, when we examined the relationships between the HR-based TLs and the session-RPE method for the different training types we found the lowest correlation for the resistance training sessions ( $r=0.25$ to 0.52 ), which typically involve short high-intensity lifting efforts. Collectively, these results suggest that there are stronger relationships between session-RPE TL and HR-based TL measures taken following endurance-based, steady-state exercise than these measures taken with stochastic, intermittent, or interval-based exercises.

Previous studies have supported the validity of the session-RPE method as a tool for quantifying internal TL for resistance training using the percentage of one-repetition maximum (1-RM) as the criterion measure of training intensity. ${ }^{7-10}$ In contrast to these previous studies, we compared the session-RPE TL method with HR-based TL methods. Since HR is considered a relatively poor method of evaluating very high intensity exercises such as resistance training, high intensity interval training, and plyometric training it seems that using HR as the criterion measure of exercise intensity is a limitation. These types of exercises depend on a large contribution from oxygen-independent metabolism rather than oxygendependent mechanisms and therefore HR may not be an appropriate global measure of exercise intensity. It is possible that other markers of exercise intensity such as blood lactate measures taken during high-intensity exercises may better relate to session-RPE measures than HR measures. ${ }^{4}$ In agreement with previous research, ${ }^{6}$ the present results suggest that the session-RPE method is a good practical method for quantifying internal TL in team sports such as soccer. 


\section{Practical Applications}

The simplicity and versatility of the session-RPE method makes it a valuable tool for athletes, coaches, and sport scientists. Its low cost and lack of reliance on technical expertise or equipment make it a very user friendly and practical tool for monitoring TL in soccer. One of the benefits of using Borg's CR-10 scale ${ }^{19}$ is its measure of both psychological and physiological factors, therefore giving a more holistic indication of the "global" internal (or physiological) stress. ${ }^{20}$ This study also supports the benefits of using the session-RPE method to monitor each player within a soccer team. Indeed, long-term monitoring of training loads may assist soccer coaches in controlling the training process and assist in improving performance.

\section{Summary}

The purpose of this study was to compare the session-RPE method with various HR-based methods for quantifying internal TL with women soccer players in a variety of training modes. The results demonstrated significant correlations between the session-RPE method and various HR-based methods for quantifying TL for all individual players. Notably, however, there was a poorer correlation with the session-RPE TL and HR-based TL during resistance training and match play. These lower relationships might be explained by the intermittent nature or the very high intensity of these activities. These results support previous findings that session-RPE is a practical method for assessing internal TL for soccer players and in particular women soccer players. Importantly, however, these results also demonstrate that session-RPE TL is not a valid substitute for HR derived TLs when monitoring exercise intensity.

\section{Acknowledgments}

Physiological test were completed at the physiology laboratory at the School of Sport and Exercise Science, Loughborough University, UK.

\section{References}

1. Impellizzeri FM, Rampinini E, Marcora SM. Physiological assessment of aerobic training in soccer $J$ Sport Sci. 2005;23(6):583-592.

2. Hoff J, Wisløff U, Engen LC, Kemi OJ, Helgerud J. Soccer specific aerobic endurance training. Br J Sports Med. 2002;36(3):218-221.

3. Foster C, Hector LL, Welsh R, Schrager M, Green MA, Snyder AC. Effects of specific versus cross-training on running performance. Eur J Appl Physiol Occup Physiol. 1995;70(4):367-372.

4. Coutts AJ, Rampinini E, Castagna C, Marcora S, Impellizzeri FM. Physiological correlates of perceived exertion during soccer-specific exercise. J Sci Med Sport. 2007:doi:10.1016/jsams.2007.1008.1005. 
5. Åstrand PO, Rodahl K. Textbook of Work Physiology. New York: McGraw Hill; 1986.

6. Impellizzeri FM, Rampinini E, Coutts AJ, Sassi A, Marcora SM. Use of RPE-based training load in soccer. Med Sci Sports Exerc. 2004;36(6):1042-1047.

7. Foster C, Florhaug JA, Franklin J, et al. A new approach to monitoring exercise training. J Strength Cond Res. 2001;15(1):109-115.

8. Day ML, McGuigan MR, Brice G, Foster C. Monitoring exercise intensity during resistance training using the session-RPE scale. J Strength Cond Res. 2004;18(2):353-358.

9. Gearhart RE, Goss FL, Lagally KM, Jakicic JM, Gallagher J, Robertson RJ. Standardized scaling procedures for rating perceived exertion during resistance exercise. $J$ Strength Cond Res. 2001;15(3):320-325.

10. Singh F, Foster C, Tod D, McGuigan MR. Monitoring different types of resistance training using session rating of perceived exertion. Int J Sport Phys Perf. 2007;2(2):34-45.

11. Borg GAV, Hassmen P, Langerstrom M. Perceived exertion in relation to heart rate and blood lactate during arm and leg exercise. Eur J Appl Physiol. 1985;65:679-685.

12. Banister EW. Modeling elite athletic performance. In: Green HJ, McDougal JD, Wenger HA, eds. Physiological Testing of Elite Athletes. Champaign, Illinois: Human Kinetics; 1991:403-424.

13. Morton RH, Fitz-Clarke JR, Banister EW. Modeling human performance in running. J Appl Physiol. 1990;69(3):1171-1177.

14. Edwards S. High performance training and racing. In: Edwards S, ed. The Heart Rate Monitor Book. 8th ed. Sacramento, CA: Feet Fleet Press; 1993:113-123.

15. Newell J, Higgins D, Madden N, et al. Software for calculating blood lactate endurance markers. J Sports Sci. 2007;25(12):1403-1409.

16. Taylor HL, Buskirk E, Henschel A. Maximal oxygen intake as an objective measure of cardio-respiratory performance. J Appl Physiol. 1955;8(1):73-80.

17. Howley ET, Bassett DR, Jr, Welch HG. Criteria for maximal oxygen uptake: review and commentary. Med Sci Sports Exerc. 1995;27(9):1292-1301.

18. Foster C. Monitoring training in athletes with reference to overtraining syndrome. Med Sci Sports Exerc. 1998;30(7):1164-1168.

19. Borg G, Ljunggren G, Ceci R. The increase of perceived exertion, aches and pain in the legs, heart rate and blood lactate during exercise on a bicycle ergometer. Eur $J$ Appl Physiol Occup Physiol. 1985;54(4):343-349.

20. Morgan WP. Psychological components of effort sense. Med Sci Sports Exerc. 1994;26(9):1071-1077. 
Copyright of International Journal of Sports Physiology \& Performance is the property of Human Kinetics Publishers. Inc. and its content may not be copied or emailed to multiple sites or posted to a listserv without the copyright holder's express written permission. However, users may print. download, or email articles for individual use. 